\title{
Acute Adverse Effects of Metallic Nanomaterials on Cardiac and Behavioral Changes in Daphnia Magna
}

\author{
Jihoon Park
}

National Institute of Chemical Safety

Chang Gyun Park

KIST Europe

\section{Baeckkyoung Sung}

KIST Europe

\section{Yong Oh Lee}

KIST Europe

Chang Seon Ryu

KIST Europe

Ja Young Park ( $\sim$ pjy0404@gmail.com )

KIST Europe

Young Jun Kim

KIST Europe

\section{Research Article}

Keywords: nanoparticle, toxicity, water flee, heart rate, swimming speed, oxidative stress

Posted Date: February 22nd, 2021

DOI: https://doi.org/10.21203/rs.3.rs-208731/v1

License: (c) (1) This work is licensed under a Creative Commons Attribution 4.0 International License.

Read Full License

Version of Record: A version of this preprint was published at Environments on February 10th, 2022. See the published version at https://doi.org/10.3390/environments9020026. 


\section{Abstract}

Nanomaterials are widely believed to induce toxic effects on organisms by evoking oxidative stress. In this study, we evaluated the toxic effects of nanomaterials on cardiac and behavioral changes in Daphnia magna under varying exposure conditions. Titanium dioxide nanoparticles $\left(\mathrm{TiO}_{2} \mathrm{NPs}\right)$, silver nanoparticles (AgNPs), and silver nitrate $\left(\mathrm{AgNO}_{3}\right)$ were selected for the acute toxicity tests. The adverse effects of the substances on the neonates including heart rate, swimming speed, and oxidative stress, were measured. The heart rate level decreased as the concentration of NPs and silver ions $\left(\mathrm{Ag}^{+}\right)$ increased. The average swimming speed was measured to be approximately $15 \mathrm{~mm} / \mathrm{min}$ for the control group. The swimming speed generally increased for longer exposure to both NPs, although it reached a plateau at the lowest concentration of AgNPs. A similar but less clear trend was observed for $\mathrm{Ag}^{+}$. For all substances, the overall swimming speed exhibited no correlation or weak negative correlations with the exposure concentration. The oxidative stress levels increased after exposure compared to the control group. We conclude that aquatic nanotoxicity tests should consider multilevel physicochemical, physiological, and behavioral parameters for the official guidelines to quantify more robust adverse outcomes.

\section{Introduction}

Nanomaterials are used in a wide range of sectors due to their specific physicochemical properties compared to their bulk materials which have the same chemical composition ${ }^{1-3}$. To ensure safe use, it is important to identify and manage the effects of exposure to nanomaterials on human and environmental health more effectively. In particular, the aquatic phase is considered a key starting point of potential entry and diffusion into the environment for understanding environmental fate and behavior. The aquatic phase creates connections with other environmental compartments such as soil, sediment, and air ${ }^{4}$.

Several studies indicate that nanomaterials are toxic to aquatic organisms such as fish and crustaceans $^{5-7}$. Of these, fishes have a direct impact on human health, but it is difficult to obtain information on the exact lethal concentration of nanomaterials from fish. Therefore, the impact of nanomaterials on non-target organisms will play an important role ${ }^{8}$. Aquatic invertebrates are a very large group of animals and some have over 1000 different types of species. Among them, arthropods are the largest invertebrate, consisting of the two largest groups: insects and crustaceans ${ }^{9}$. Crustaceans are the most important group of invertebrates, of which Daphnia magna is commonly used to assess the risk of nanomaterials ${ }^{10}$. Algae and crustaceans exposed to nanomaterials can poison and cause death to aquatic organisms such as fish through the food chain ${ }^{5,11}$.

Nanomaterials are generally known to have higher toxicity than bulk materials. It has been reported from in vitro studies that nanomaterials can induce biological effects such as cell inflammation and apoptosis by oxidative stress ${ }^{12-15}$. The preliminary factors causing toxicity are size, shape, and surface area/charge ${ }^{16}$. Nanomaterials are more reactive due to their large surface area, and easy penetration into 
cells $^{16,17}$. Depending on the surface properties, the nanomaterials may exhibit enhanced interactions with the cell membranes ${ }^{18,19}$.

Here, we focus on two representative types of metallic nanoparticles, titanium dioxide $\left(\mathrm{TiO}_{2}\right)$ and sliver (Ag) nanoparticles (NPs). $\mathrm{TiO}_{2} \mathrm{NPs}$ are generally known to be non-toxic and can be applied in various ways such as through cosmetics and catalysts ${ }^{20,21}$. AgNPs are also used in a wide range of consumer products as antibacterial agents due to their high cytotoxicity ${ }^{22-24}$. One of the most fundamental sources of AgNP toxicity has been attributed to the release of sliver ions $\left(\mathrm{Ag}^{+}\right)$in tissue environments.

Daphnia magna is a standard organism for ecotoxicity test using toxic chemicals in the standardized protocols suggested by the Organization for Economic Cooperation and Development (OECD), International Organization for Standardization (ISO), and the United States Environmental Protection Agency (USEPA) ${ }^{25,26}$. As an organism in the bottom stage of the food chain, $D$. magna plays a key role in aquatic ecosystems since it is sensitive to environmental stresses, and population change can ultimately affect the population of upper predators ${ }^{25,27}$. Previous studies have reported the biological effects of hazardous chemicals on physiological factors such as cardiac and behavioral characteristics in $D$. magna ${ }^{28-32}$. Fekete-Kertész et al. (2017) evaluated the change in heart rate using $D$. manga and found that heart rate was influenced by several factors; chemical exposure level (triclosan), test medium, organism age, and exposure time ${ }^{33}$. Chung et al. (2016) also reported that the heart rate and swimming distance were linearly changed by $\mathrm{TiO}_{2}$ nanoparticle concentration ${ }^{34}$. In particular, they adopted a video tracking method which has been recently used to evaluate the behavioral characteristics of organisms such as zebrafish and $D$. magna. Therefore, we aimed to evaluate the toxic effects on the physiological factors of $D$. manga using nanomaterials and to identify the relationships between the factors according to the exposure conditions in this study.

\section{Results}

\section{Effects of nanomaterials on the heart rate}

Immobilization and heart rate were measured after acute exposure. The acute immobilization test was performed in accordance with OECD guidelines 202. A median lethal concentration ( $\left.\mathrm{LC}_{50}\right)$ value of $\mathrm{TiO}_{2}$ in D. magna after 48 hours was determined to be more than $100 \mathrm{mg} / \mathrm{ml}$ and the $L_{50}$ values of AgNPs and $\mathrm{Ag}^{+}$were 0.0134 and $0.0016 \mu \mathrm{g} / \mathrm{ml}$, respectively (Fig. 1).

The heart rate decreased as the concentration of all substances $\left(\mathrm{TiO}_{2} \mathrm{NPs}, \mathrm{AgNPs}, \mathrm{Ag}^{+}\right)$increased. The heart rate of the control group was observed to $364 \pm 28$ BPM (beats per minute). At the lowest concentration level of each substance after exposure for 3 and 48 hours (355-385 BPM), the heart rate was not significantly different from that of the control level, but it decreased by approximately $7.3 \%\left(\mathrm{TiO}_{2}\right.$, $345 \pm 17 \mathrm{BPM}), 4.3 \%$ (AgNP, $347 \pm 26 \mathrm{BPM})$, and 15.0\% ( $\left.\mathrm{Ag}^{+}, 302 \pm 15 \mathrm{BPM}\right)$ at the highest concentration level after exposure for 3 hours, respectively (Fig. 2A). After 48 hours of exposure, the heart rate recovered 
at the lowest and medium concentration levels compared to the control level. At the lowest concentration of each substance, the heart rate was $378 \pm 25 \mathrm{BPM}$ for $\mathrm{TiO}_{2}$ NPs, $385 \pm 14 \mathrm{BPM}$ for AgNPs, and $375 \pm 17$ $\mathrm{BPM}$ for $\mathrm{Ag}^{+}$, but it gradually decreased with increasing concentration level for all substances (Fig. 2B). The reduction rate with a higher concentration was relatively lower than that after 3 hours of exposure $\left(\mathrm{TiO}_{2} \mathrm{NPs}, 5.8 \% ; \mathrm{AgNPs}, 2.4 \% ; \mathrm{Ag}^{+}, 3.3 \%\right)$.

\section{Effects on the behavioral performance}

Behavioral performance was measured as the swimming speed, which is an averaged moving distance per minute and the speed level of the control group was approximately $15 \mathrm{~mm} / \mathrm{min}$. Real-time tracking paths were recorded according to the instantaneous swimming speed as shown in Fig. 3 with examples of 10-minute exposure. The observed behavioral change upon substance exposure was limited to locomotive movements. The swimming speed generally increased for longer exposure to both NPs, although it reached a plateau at the lowest concentration of AgNPs. A similar but less clear trend was observed for $\mathrm{Ag}^{+}$. The swimming behavior appeared to be more activated at the lowest concentration level of each substance compared to higher concentration levels. For the neonates that were exposed to $0.1 \mu \mathrm{g} / \mathrm{mL} \mathrm{TiO}_{2} \mathrm{NPs}$, the swimming speed increased proportionally with the exposure duration (from 8.2 $\pm 0.9 \mathrm{~mm} / \mathrm{min}$ for 3 hours to $21.6 \pm 2.8 \mathrm{~mm} / \mathrm{min}$ for 48 hours). In addition, the swimming speed increased with increasing exposure duration, although the swimming speed was lower at higher concentrations (1.0 and $10 \mu \mathrm{g} / \mathrm{mL}$ ) than at the lowest level $(0.1 \mu \mathrm{g} / \mathrm{mL})$ (Fig. 4A). In the case of AgNPs, the swimming speed showed irregular patterns by the concentration level and exposure duration. It significantly increased up to the 2-hour exposure duration from 7.4 to $17.2 \mathrm{~mm} / \mathrm{min}$, and decreased slightly after 24-hour exposure at $0.0001 \mu \mathrm{g} / \mathrm{mL}$. It continuously increased in proportion to the exposure duration at $0.001 \mu \mathrm{g} / \mathrm{mL}$ from 6.7 to $16.0 \mathrm{~mm} / \mathrm{min}$, but this trend was weakened at the highest concentration (Fig. 4B). At the lowest concentration of $\mathrm{Ag}^{+}(0.00001 \mu \mathrm{g} / \mathrm{mL})$, the speed increased proportionally up to 24 hours of exposure (from $7.0 \pm 0.8 \mathrm{~mm} / \mathrm{min}$ for 3 hours to $21.7 \pm 4.1 \mathrm{~mm} / \mathrm{min}$ for 24 hours). There was no obvious pattern at higher concentration levels, but the speed peaked for the neonates exposed for 24 hours (Fig. 4C). Realtime temporal variations in swimming speed for each material are available in the supplementary information (Fig. A1-A3).

\section{Quantification of oxidative stress}

Reactive oxygen stress (ROS) levels were measured by 2,7-dichlorofluorescein diacetate (DCFDA) assay. Figures $5 \& 6$ show the results of ROS measurements in $D$. magna. All three materials exhibited stronger intensities than the control group after 3 hours of exposure. The error bars in Fig. 6 represent the arithmetic mean and standard deviation $(n=3$, mean $\pm S D)$. In particular, the ROS intensity of $\mathrm{TiO}_{2}$ significantly increased in proportion to the concentration, but the AgNPs and their ions showed concentration-dependent tendencies and then decreased at the highest concentration $(0.01 \mu \mathrm{g} / \mathrm{mL}$ for AgNPs and $0.001 \mu \mathrm{g} / \mathrm{mL}$ for $\mathrm{Ag}^{+}$). The results indicate that exposure to all substances for 3 hours significantly affected ROS levels, while exposure for 48 hours decreased ROS levels at a higher 
concentration, which is likely due to the alteration of the effective concentration after 48 hours of exposure.

\section{Discussion}

The toxicity test using D. magna is well documented in the OECD guidelines 202 and it is determined by immobilization at 24 and 48 hours after exposure to nanoparticles. In most cases, it is difficult to determine accurate toxic effects based on the method because the data varies depending on the different groups. Considering this limitation, we tried to test the acute toxicity on D. magna using influential indices including immobilization, heart rate, swimming performance and ROS level.

First, we determined the $\mathrm{LC}_{50}$ values for each material as the first step in the exposure test using organisms. The $\mathrm{LC}_{50}$ value of $\mathrm{TiO}_{2}$ in $D$. magna after 48 hours was determined to be more than 100 $\mathrm{mg} / \mathrm{ml}$ and the values of AgNPs and $\mathrm{Ag}^{+}$were 0.0134 and $0.0016 \mu \mathrm{g} / \mathrm{ml}$, respectively. Ribeiro et al. (2014) determined the $\mathrm{LC}_{50}$ of AgNPs to be $11.02 \mu \mathrm{g} / \mathrm{L}$ after 48 hours of exposure, and $1.05 \mu \mathrm{g} / \mathrm{L}$ for silver nitrate $\left(\mathrm{AgNO}_{3}\right)$ using $D$. magna ${ }^{35}$. Shen et al. (2015) also calculated $\mathrm{LC}_{50}$ values of $0.58-2.51 \mu \mathrm{g} / \mathrm{L}$ for $\mathrm{AgNO}_{3}$, which were lower than our results ${ }^{36}$. The results occur depending on the strain and water physicochemical parameters, which means that even if we measured the $\mathrm{LC}_{50}$ considering experimental conditions, the outcomes might be different between laboratories.

The change in heart rate as an index of toxic effects on $D$. magna is widely used in acute toxicity tests. The heart rate was measured repeatedly using more than 10 neonates exposed to the materials and a cross check for all measurements was also performed to minimize bias by observers. The results showed that the heart rate decreased with increasing concentration for all substances. It was possible to obtain a similar pattern even for 3-hour exposure with the OECD guidelines (48-hour exposure) ${ }^{37}$. Thus, we confirmed that an exposure duration of 3 hours was sufficient to test acute toxicity. In addition, $\mathrm{TiO}_{2} \mathrm{NPs}$ considered to be non-toxic, also reduced the heart rate by increasing the exposure level. This showed that $\mathrm{TiO}_{2}$ NPs affect the heart rate of $D$. magna, similar to other toxic NPs (e.g., AgNPs), but it is limited to conclude that $\mathrm{TiO}_{2} \mathrm{NPs}$ influence the immobilization (behavioral performances).

Locomotion-based behavior is a highly sensitive index for identifying toxic effects of chemicals ${ }^{28,38}$. Thus, immobilization was characterized by measuring swimming speed (video tracking) in addition to heart rate counting. In particular, the behavioral change was measured at each exposure duration $(3,6$, $12,24,48$ hours), thus we could identify the temporal effects in detail compared to most previous studies that examined tests after 48 hours of exposure according to the OECD guidelines 202 (Fig. A1-A3 in the Supplementary Information).

To date, information on not only the temporal variations in immobilization but also the relationship between heart rate and behavioral responses to the toxic effects of nanoparticles remains limited. For example, Lovern et al. (2007) examined behavioural and physiological changes in $D$. magna using $\mathrm{TiO}_{2}$ 
NPs, fullerenes and fullerene derivatives ${ }^{31}$. The authors found that an increased exposure level influenced the low heart rate and movement. Additionally, the effect was not statistically significant for $D$. manga exposed to $\mathrm{TiO}_{2} \mathrm{NPs}$, but the temporal variation was not characterized. In our study, the heart rate increased after 48 hours of exposure compared with that after 3 hours of exposure, while it decreased in proportion to the exposure level (Fig. 2). The swimming speed at an earlier exposure duration (3 hours) was low for all materials and increased as time elapsed (Fig. 4). Therefore, it can be inferred that the exposure duration has more influences on the cardiac and behavioral effects at earlier periods, and $D$. magna are more active after a certain stabilization period.

The ROS level was additionally measured to support the outcomes in this study. The ROS levels of the target materials increased with the exposure level, but they gradually decreased from a certain level after 48 hours of exposure. The relative intensities of ROS were observed with inverted U-shaped patterns after 48 hours of exposure. This can be interpreted as the ROS levels recovered by enhanced antioxidative responses at high concentrations ${ }^{39,40}$ and the overall fluorescence is enhanced by ROS production from the nanoparticles. The excessive ROS generation can cause oxidative stress ${ }^{41}$. Previous studies have also reported that oxidative stress can cause toxic effects on heart rate, swimming speed, and reproduction in D. magna ${ }^{42-44}$. In this study, it was observed that the nanoparticles enhanced the ROS levels compared to the control group, which is also consistent with previous studies ${ }^{13,45}$.

There have been significant differences between the results on immobilization, cardiac effects and behavioral changes in previous studies. Therefore, a comprehensive test including these indices would be necessary to improve the quality of the results on acute toxicity. Furthermore, the effects of nanomaterial mixtures on aquatic toxicity need to be explored in terms of invertebrate heart physiology and swimming patterns ${ }^{46,47}$.

\section{Methods}

\section{Experimental scheme}

This study consists of three steps to examine the toxicity of nanomaterials using $D$. magna. The procedures include sample preparation (test organism, test substances), treatment (exposure), and analytical tests using instruments. One-day-old neonates that were newly born within 24 hours were prepared and certain exposure levels of nanomaterials were treated for different exposure times. After

exposure duration at each concentration level, physiological effects on the neonates including the cardiac effect and behavioral changes were characterized. The test procedures for each process are described in the following sections in detail.

\section{Daphnia magna culture}

D. magna cultured in the laboratory was used in a series of experiments. The test organism was cultured in $5 \mathrm{~L}$ beakers in a $21 \pm 1^{\circ} \mathrm{C}$ thermostatic incubator under a constant light cycle for 24 hours. The light 
conditions were maintained in the light for 16 hours, and then the light was turned off for 8 hours ( $12 \mathrm{~W}$, $356 \mathrm{~mm}$ lamp, 672 Lumen, $4200 \mathrm{~K}$ ). To maintain the breeding condition, the $D$. magna adults were cultivated in OECD M4 medium, and daily feeding for the organisms was conducted using an algae containing a mixture of Chlorella vulgaris and additional nutrition (yeast, cerophyll and trout chow; $Y C T)^{48}$. One-day-old neonates of $D$. magna were used for the exposure test. To check the sensitivity of the $D$. magna culture, an acute immobilization toxicity test was performed with potassium dichromate ( $\mathrm{K} 2 \mathrm{Cr} 207)$ as a reference. The sensitivity of $D$. magna culture to $\mathrm{K} 2 \mathrm{Cr} 2 \mathrm{O} 7$ ranged within the limits (halfmaximal effective dose, $\mathrm{EC}_{50}: 0.6-2.1 \mathrm{mg} / \mathrm{L}$ for 24 hours) suggested by OECD guideline 202. For each test concentration and control, five neonates each ( $<15$ hours-old) were placed in 6-well plates (VWR Tissue Culture Plates, VWR, Darmstadt, Germany) containing $10 \mathrm{~mL}$ of either test solution or suspension. The neonates ( $<24$ hours-old) obtained from the fifth generation were used in the toxicity tests to minimize variability. After incubation for 3 and 48 hours, $D$. magna was used to measure the heart rate and swimming speed.

\section{Preparation of nanomaterials}

A total of three commercially available materials including two metallic nanoparticles $\left(\mathrm{TiO}_{2} \mathrm{NPs}\right.$, AgNPs) and $\mathrm{AgNO}_{3}$ were used for tests in this study. The metallic nanomaterials were purchased from manufacturers $\left(\mathrm{TiO}_{2}\right.$ : Aeroxide ${ }^{\circledR} \mathrm{TiO}_{2} \mathrm{P} 25$, Evonik Industries, Germany; AgNP: NanoXactTM, NanoComposix Inc., USA) and the particle size of each nanomaterial was measured using a transmission electron microscope (TEM). Individual $\mathrm{TiO}_{2}$ particles are normally $21 \mathrm{~nm}$ in size, and their aggregates are distributed hundreds of nanometers in size. Spherical AgNPs were provided at $0.02 \mathrm{mg} / \mathrm{mL}$ in a $2 \mathrm{mM}$ sodium citrate solution, and the primary particle size was $31 \pm 3 \mathrm{~nm}$. Silver nitrate (ACS reagent, purity $\geq$ 99.0\%; Sigma-Aldrich, St. Louis, MO, United States) was used as a source of $\mathrm{Ag}^{+}$. All the chemicals were used without further purification.

\section{Determination of exposure levels}

The $\mathrm{TiO}_{2}$ NP stock solutions were made by dispersing $1 \mathrm{mg}$ in $1 \mathrm{ml}$ of distilled water and then sonicating for $30 \mathrm{~min}$. Different suspended concentrations were prepared using ISO medium for each material. The OECD-recommended ISO medium consisted of a mixture of $294 \mathrm{mg} / \mathrm{L} \mathrm{CaCl} \cdot 2 \mathrm{H}_{2} \mathrm{O}, 123.25 \mathrm{mg} / \mathrm{L}$ $\mathrm{MgSO}_{4} \cdot 7 \mathrm{H}_{2} \mathrm{O}, 64.75 \mathrm{mg} / \mathrm{L} \mathrm{NaHCO}_{3}, 5.75 \mathrm{mg} / \mathrm{L} \mathrm{KCl}$, and $2 \mu \mathrm{g} / \mathrm{L} \mathrm{Na}_{2} \mathrm{SeO}_{3} 44$. The diluted solutions of $\mathrm{TiO}_{2}$ NPs were prepared with concentrations of $0.1,1.0$, and $10 \mu \mathrm{g} / \mathrm{mL}$. The concentration levels of AgNPs and silver ions were $0.0001,0.001,0.01 \mu \mathrm{g} / \mathrm{mL}$ and $0.00001,0.00010 .001 \mu \mathrm{g} / \mathrm{mL}$, respectively. All diluted suspensions were immediately homogenized after vortexing. Each concentration range was determined with reference to the $\mathrm{EC}_{50}$ from previous studies $25,35,49-58$.

After each material was dispersed in the medium, the size distribution of each material was measured using a TEM (JEM-2100 LaB 6 ; JEOL, Tokyo, Japan) to identify whether the particle size was changed by medium type (distilled water vs. ISO medium) (Fig. 7). Characterization of the NP suspensions was conducted under a $200 \mathrm{kV}$ accelerating voltage. An aliquot of each suspension was rinsed over a holey 
carbon TEM grid (type S147-4, Plano, Wetzlar, Germany) and then dried at room temperature. More than 100 particles were taken at three magnifications due to the different particle sizes. The images were analyzed for average length (diameter) using the pixel ruler via Image $\mathrm{J}$ software. The primary particle size of $\mathrm{TiO}_{2}$ was $24 \pm 5 \mathrm{~nm}$ and the AgNP was $31 \pm 3 \mathrm{~nm}$ (Fig. 7A \& 7C). However, we found that the primary particles aggregated in the medium over 48 hours (Fig. 7B \& 7D). Thus, we confirmed that $D$. magna can be exposed to primary and aggregated particles simultaneously.

\section{Heart rate counting}

The most commonly used endpoint of toxicity is to measure the death rates of $D$. magna. The immobilization of $D$. magna immobilization was observed at each concentration before counting the heart rate. $L_{50}$ is defined as the concentration of toxic substances that kill $50 \%$ of the test organism within a certain exposure period. Survival data were plotted and $\mathrm{LC}_{50}$ values were calculated using logistic 3-parameter curve fitting with Sigmaplot 13.0 software (Systat Software Inc., San Jose, CA, USA).

The heart rate was measured to evaluate the influences of exposure to each nanomaterial in triplicate. Neonates hatched within 24 hours from the fifth generation of $D$. manga adults were newly prepared on the day of the experiment. Every five neonates were exposed to each concentration level for 3 and 48 hours. The control group was also prepared for comparison with exposure groups under the same conditions without chemical treatment. After exposure, an aliquot of the methyl cellulose solution ( $4 \%$ V/W, Lot No. SLCC9072, Sigma-Aldrich Corp., St. Louis, MO, United States) was used to fix individual neonates on glass plate. We observed heart rate conditions for one minute using an optical microscope at 4X magnification (Model CKX41, Olympus Inc., Tokyo, Japan), and recorded them with video files. The heart rate was finally counted manually in the play condition of low speed $(\times 0.3)$.

\section{Swimming performance monitoring}

As a behavioral index, the swimming performance of $D$. magna after exposure to each concentration level of nanomaterials was measured using a direct-reading instrument (Model Zebrabox, View Point Life Science Inc., Lyon, France). Individual neonates exposed to each concentration were transferred to each well of a 96-well plate separately. Each well plate batch was placed into the Zebrabox and all exposure groups were kept stable in entirely dark conditions for 30 minutes before measurement to minimize the effects of sudden environmental changes. After stabilization for $30 \mathrm{~min}$, the swimming speed (distance per minute) and moving route were measured under tracking mode every minute for 80 minutes. The realtime data were analyzed using automated observation software (Zebralab-2, View Point Life Science Inc., Lyon, France).

\section{Measurement of oxidative stress}

The measurement of ROS in D. magna was measured using a 2, 7-dichlorofluorescein diacetate (DCFDA) cellular ROS detection assay kit (ab113851, Abcam, Berlin, Germany). After nanoparticle exposure for 3 and 48 hours, 10 for each concentration of $D$. magna were washed in a beaker with pure water and 
transferred to Eppendorf tubes with $200 \mu \mathrm{L}$ of phosphate buffered saline (PBS). D. magna was homogenized by VWR® Disposable Pellet Mixers and Cordless Motor (VWR, Darmstadt, Germany). Then, the homogenates were centrifuged at $13,000 \mathrm{xg}$ for 20 minutes, and the supernatant of samples was collected. Samples were kept at $-80^{\circ} \mathrm{C}$ until the assay was performed. Twenty microliters of each collected supernatant and $80 \mu \mathrm{L}$ of assay buffer were placed on a black 96 -well microplate (ThermoScientific, Karlsruhe, Germany) and treated with $100 \mu \mathrm{L}$ of $10 \mu \mathrm{M}$ DCFDA. Then, fluorescence measurements were conducted immediately after incubation for 30 minutes in the dark using Spark $\AA$ Multimode Microplate Reader (Tecan Trading AG, Männedorf, Switzerland). The excitation state was maintained at a wavelength of $485 \mathrm{~nm}$, and the emission state was maintained at a wavelength of 535 $\mathrm{nm}$. Each concentration was measured five times and the mean value was obtained. The total protein content of each sample was quantified by a BCA protein assay for the normalization of samples. The DCFDA levels in the $D$. magna were also visualized using a Zeiss SteREO Discovery V8 microscope with a Plan S 1.0xFWD $81 \mathrm{~mm}$ objective (Carl Zeiss NTS, Ltd., Jena, Germany). After that, the captured images were analyzed using ZEN imaging software (Carl Zeiss NTS Ltd., Jena, Germany).

\section{Declarations}

\section{Acknowledgements}

This work was supported by the Basic Science Research Program (Grant No. 2019R1A6A3A03033990) through the National Research Foundation (NRF) funded by the Ministry of Education, and by the Nanomaterial Technology Development Program (Grant No. 2017M3A7B6052455) funded by the Ministry of Science \& ICT, and by the Technology Innovation Program (Grant No. 20011630) funded by the Ministry of Trade, Industry \& Energy. We do appreciate to Dr. Marcus Koch (INM, Saarbrücken, Germany) for TEM imaging.

\section{Author contributions}

J. Park: Data Curation, Formal Analysis, Investigation, Validation, Visualization, Writing-Original Draft Preparation, Writing-Review \& Editing; C.G. Park: Data Curation, Formal Analysis, Investigation, Validation, Writing-Original Draft Preparation; B. Sung: Conceptualization, Validation, Writing-Review \& Editing; Y.O. Lee: Conceptualization, Validation, Writing-Review \& Editing; C.S. Ryu: Conceptualization, Validation, Writing-Review \& Editing; Y.J. Kim: Conceptualization, Supervision, Validation, Writing-Review \& Editing; J. Park (corresponding author): Data Curation, Formal Analysis, Investigation, Validation, Writing-Original Draft Preparation, Writing-Review \& Editing.

\section{Competing interests}

The authors declare there is no conflict of interest.

\section{References}


1. Aschberger, K. et al. Considerations on information needs for nanomaterials in consumer products. Preprint at https://iacmcolor.org/wp-content/uploads/2014/08/JRC-Consumer-Product-Labeling-fornano-Policy-Report-0414.pdf (2014).

2. Boverhof, D. R. \& David, R. M. Nanomaterial characterization: considerations and needs for hazard assessment and safety evaluation. Anal. Bioanal. Chem. 396, 953-961 (2010).

3. Oomen, A., Bennink, M., van Engelen, J. \& Sips, A. Nanomaterial in consumer products: Detection, characterisation and interpretation. Preprint at https://rivm.openrepository.com/handle/10029/257129 (2011).

4. Hartmann, N. I. B. et al. Environmental fate and behaviour of nanomaterials: new knowledge on important transfomation processes. The Danish Environmental Protection Agency. Environmental Project No. 1594(2014).

5. Cedervall, T., Hansson, L. A., Lard, M., Frohm, B. \& Linse, S. Food chain transport of nanoparticles affects behaviour and fat metabolism in fish. PloS One. 7, e32254 (2012).

6. Hou, J. et al. Molecular toxicity of metal oxide nanoparticles in Danio rerio. Environ. Sci. Technol. 52, 7996-8004 (2018).

7. Lu, G., Yang, H., Xia, J., Zong, Y. \& Liu, J. Toxicity of Cu and Cr nanoparticles to Daphnia magna. Water Air Soil Pollut. 228, 18 (2017).

8. De Marchi, L. et al. The impacts of seawater acidification on Ruditapes philippinarum sensitivity to carbon nanoparticles. Environ. Sci. Nano. 4, 1692-1704 (2017).

9. Ruppert, E. E., Barnes, R. D. \& Fox, R. S. Invertebrate zoology: a functional evolutionary approach. (ed. Brooks Cole)(Cengage Learning 7th edition, 2003).

10. European Chemicals Bureau. Technical Guidance Document on Risk Assessment-Part 1. Preprint at https://echa.europa.eu/documents/10162/16960216/tgdpart1_2ed_en.pdf. (2003).

11. Tangaa, S. R., Selck, H., Winther-Nielsen, M. \& Khan, F. R. Trophic transfer of metal-based nanoparticles in aquatic environments: a review and recommendations for future research focus. Environ. Sci. Nano. 3, 966-981 (2016).

12. Fu, P. P., Xia, Q., Hwang, H. M., Ray, P. C. \& Yu, H. Mechanisms of nanotoxicity: generation of reactive oxygen species. J. Food Drug Anal. 22, 64-75 (2014).

13. Kim, T. H. et al. Size-dependent cellular toxicity of silver nanoparticles. J. Biomed. Mater. Res. A. 100, 1033-1043 (2012).

14. Miller, M. R. Oxidative stress and the cardiovascular effects of air pollution. Free Radical Bio. Med. 151, 69-87 (2020).

15. Xia, T., Li, N. \& Nel, A. E. Potential health impact of nanoparticles. Annu. Rev. Publ. Health. 30, 137150 (2009).

16. Pietroiusti, A., Campagnolo, L. \& Fadeel, B. Interactions of engineered nanoparticles with organs protected by internal biological barriers. Small. 9, 1557-1572 (2013). 
17. Pietroiusti, A. et al. Nanomaterial exposure, toxicity, and impact on human health. Wiley Interdiscip. Rev. Nanomed. Nanobiotechnol. 10, e1513 (2018).

18. Gatoo, M. A. et al. Physicochemical properties of nanomaterials: implication in associated toxic manifestations. BioMed. Res. Int. 2014, 498420 (2014).

19. Navya, P. \& Daima, H. K. Rational engineering of physicochemical properties of nanomaterials for biomedical applications with nanotoxicological perspectives. Nano Converg. 3, 1 (2016).

20. Hou, J. et al. Toxicity and mechanisms of action of titanium dioxide nanoparticles in living organisms. J. Environ. Sci. 75, 40-53 (2019).

21. Rizk, M. Z. et al. Toxicity of titanium dioxide nanoparticles: effect of dose and time on biochemical disturbance, oxidative stress and genotoxicity in mice. Biomed. Pharmacother. 90, 466-472 (2017).

22. Nomiya, K. et al. Synthesis and structural characterization of silver (I), aluminium (III) and cobalt (II) complexes with 4-isopropyltropolone (hinokitiol) showing noteworthy biological activities. Action of silver (I)-oxygen bonding complexes on the antimicrobial activities. J. Inorg. Biochem. 98, 46-60 (2004).

23. Sarmast, M., Salehi, H. \& Khosh-Khui, M. Nano silver treatment is effective in reducing bacterial contaminations of Araucaria excelsa R. Br. var. glauca explants. Acta Biol. Hung. 62, 477-484 (2011).

24. Seitz, F. et al. Effects of silver nanoparticle properties, media pH and dissolved organic matter on toxicity to Daphnia magna. Ecotox. Environ. Safe. 111, 263-270 (2015).

25. Asghari, S. et al. Toxicity of various silver nanoparticles compared to silver ions in Daphnia magna. J. Nanobiotechnol. 10, 14 (2012).

26. Hu, Y., Chen, X., Yang, K. \& Lin, D. Distinct toxicity of silver nanoparticles and silver nitrate to Daphnia magna in M4 medium and surface water. Sci. Total Environ. 618, 838-846 (2018).

27. Martins, J., Teles, L. O. \& Vasconcelos, V. Assays with Daphnia magna and Danio rerio as alert systems in aquatic toxicology. Environ. Int. 33, 414-425 (2007).

28. Bownik, A. Daphnia swimming behaviour as a biomarker in toxicity assessment: a review. Sci. Total Environ. 601, 194-205 (2017).

29. Kunze, J., Hartmann, S., Witte, K. \& Kuhnert, K. D. Daphnia magna as biosensor for Ag-nanoparticles in water systems: Development of a computer vision system for the detection of behavioral changes. Preprint at http://homepages.inf.ed.ac.uk/rbf/VAIB16PAPERS/vaibkunze.pdf (2016).

30. Lovern, S. B. \& Klaper, R. Daphnia magna mortality when exposed to titanium dioxide and fullerene (C60) nanoparticles. Environ. Toxicol. Chem. 25, 1132-1137 (2006).

31. Lovern, S. B., Strickler, J. R. \& Klaper, R. Behavioral and physiological changes in Daphnia magna when exposed to nanoparticle suspensions (titanium dioxide, nano- 660 , and $\mathrm{C} 60 \mathrm{Hx} \mathrm{C} 70 \mathrm{Hx}$ ). Environ. Sci. Technol. 41, 4465-4470 (2007).

32. Simão, F. C. et al. Using a new high-throughput video-tracking platform to assess behavioural changes in Daphnia magna exposed to neuro-active drugs. Sci. Total Environ. 662, 160-167 (2019). 
33. Fekete-Kertész, I. et al. How Does Experimental Design Modify the Result of Daphnia magna Heartbeat Rate Test? Analyses of Factors Affecting the Sensitivity of the Test System. Period. Polytech-Chem. 62, 257-264 (2018).

34. Chung, W., Song, J. M. \& Lee, J. The Evaluation of titanium dioxide nanoparticle effects on cardiac and swimming performance of Daphnia magna. Int. J. Appl. Environ. Sci. 11, 1375-1385 (2016).

35. Ribeiro, F. et al. Silver nanoparticles and silver nitrate induce high toxicity to Pseudokirchneriella subcapitata, Daphnia magna and Danio rerio. Sci. Total Environ. 466, 232-241 (2014).

36. Shen, M. H. et al. Exposure medium: key in identifying free $\mathrm{Ag}+$ as the exclusive species of silver nanoparticles with acute toxicity to Daphnia magna. Sci. Rep. 5, 9674 (2015).

37. Organization for Economic Cooperation and Development (OECD). OECD guideline for testing of chemicals - Daphnia sp., Acute immobilization test. Preprint at https://www.oecdilibrary.org/environment/test-no-202-daphnia-sp-acute-immobilisation-test_9789264069947-en (2002).

38. Mora-Zamorano, F. X., Larson, J. K. \& Carvan, M. J. Neurobehavioral analysis methods for adverse outcome pathway (AOP) models and risk assessment in A Systems Biology Approach to Advancing Adverse Outcome Pathways for Risk Assessment (ed. Garcia-Reyero, N.) 149-175(Springer, 2018).

39. Galdiero, E. et al. An integrated study on antimicrobial activity and ecotoxicity of quantum dots and quantum dots coated with the antimicrobial peptide indolicidin. Int. J. Nanomed. 11, 4199 (2016).

40. Zhou, H. et al. BI1 alleviates cardiac microvascular ischemia-reperfusion injury via modifying mitochondrial fission and inhibiting XO/ROS/F-actin pathways. J. Cell. Physiol. 234, 5056-5069 (2019).

41. Klaper, R. et al. Toxicity biomarker expression in daphnids exposed to manufactured nanoparticles: changes in toxicity with functionalization. Environ. Pollut. 157, 1152-1156 (2009).

42. Bownik, A. \& Stępniewska, Z. Protective effects of ectoine on behavioral, physiological and biochemical parameters of Daphnia magna subjected to hydrogen peroxide. Com. Biochem. Phys. C. 170, 38-49 (2015).

43. De Felice, B., Salgueiro-González, N., Castiglioni, S., Saino, N. \& Parolini, M. Biochemical and behavioral effects induced by cocaine exposure to Daphnia magna. Sci. Total Environ. $689,141-148$ (2019).

44. Liu, Z. et al. Effects of nanoplastics at predicted environmental concentration on Daphnia pulex after exposure through multiple generations.. Environ. Pollut. 256, 113506 (2020).

45. Lu, H., Fan, W., Dong, H. \& Liu, L. Dependence of the irradiation conditions and crystalline phases of TiO2 nanoparticles on their toxicity to Daphnia magna. Environ. Sci. Nano. 4, 406-414 (2017).

46. Baek, M. J. et al. Quantitative prediction of mixture toxicity of AgNO3 and $\mathrm{ZnO}$ nanoparticles on Daphnia magna. Sci. Technol. Adv. Mater. 21, 333-345 (2020).

47. Park, C. B. et al. Mixture toxicity of metal oxide nanoparticles and silver ions on Daphnia magna. J. Nanopart. Res. 21, 166 (2019). 
48. National Institute of Environmental Research (NIER). Operational guideline for ecotoxicity test procedures. Document No. NIER-GP2015-094. Preprint at https://ecolibrary.me.go.kr/nier/\#/search/detail/5595086 (in Korean) (2015).

49. Baumann, J., Sakka, Y., Bertrand, C., Köser, J. \& Filser, J. Adaptation of the Daphnia sp. acute toxicity test: miniaturization and prolongation for the testing of nanomaterials. Environ. Sci. Pollut. R. 21, 2201-2213 (2014).

50. Cupi, D., Hartmann, N. B. \& Baun, A. Influence of $\mathrm{pH}$ and media composition on suspension stability of silver, zinc oxide, and titanium dioxide nanoparticles and immobilization of Daphnia magna under guideline testing conditions. Ecotoxicol. Environ. Safe. 127, 144-152 (2016).

51. Georgantzopoulou, A. et al. Ag nanoparticles: size-and surface-dependent effects on model aquatic organisms and uptake evaluation with NanoSIMS. Nanotoxicology. 7, 1168-1178 (2012).

52. Heinlaan, M., Ivask, A., Blinova, I., Dubourguier, H. C. \& Kahru, A. Toxicity of nanosized and bulk ZnO, $\mathrm{CuO}$ and $\mathrm{TiO} 2$ to bacteria Vibrio fischeri and crustaceans Daphnia magna and Thamnocephalus platyurus. Chemosphere. 71, 1308-1316 (2008).

53. Hlavkova, D., Havelkova, B., Kopel, P. \& Beklova, M. Evaluation of nanosilver ecotoxicity using representatives of distinct trophic levels. Fresenius Environ. Bull. 28, 745-749 (2019).

54. Hurel, C. et al. Functionalized and grafted $\mathrm{TiO} 2, \mathrm{CeO} 2$, and $\mathrm{SiO} 2$ nanoparticles-ecotoxicity on Daphnia magna and relevance of ecofriendly polymeric networks. Environ. Sci. Pollut. R. 25, 2121621223 (2018).

55. Jemec, A. et al. An interlaboratory comparison of nanosilver characterisation and hazard identification: Harmonising techniques for high quality data. Environ. Int. 87, 20-32 (2016).

56. Kim, J., Kim, S. \& Lee, S. Differentiation of the toxicities of silver nanoparticles and silver ions to the Japanese medaka (Oryzias latipes) and the cladoceran Daphnia magna. Nanotoxicology. 5, 208214 (2011).

57. Marcone, G. P., Oliveira, Ã. C., Almeida, G., Umbuzeiro, G. A. \& Jardim, W. F. Ecotoxicity of TiO2 to Daphnia similis under irradiation. J. Hazard. Mater. 211, 436-442 (2012).

58. Picado, A. et al. A multi-integrated approach on toxicity effects of engineered TiO2 nanoparticles. Front. Env. Sci. Eng. 9, 793-803 (2015).

\section{Figures}




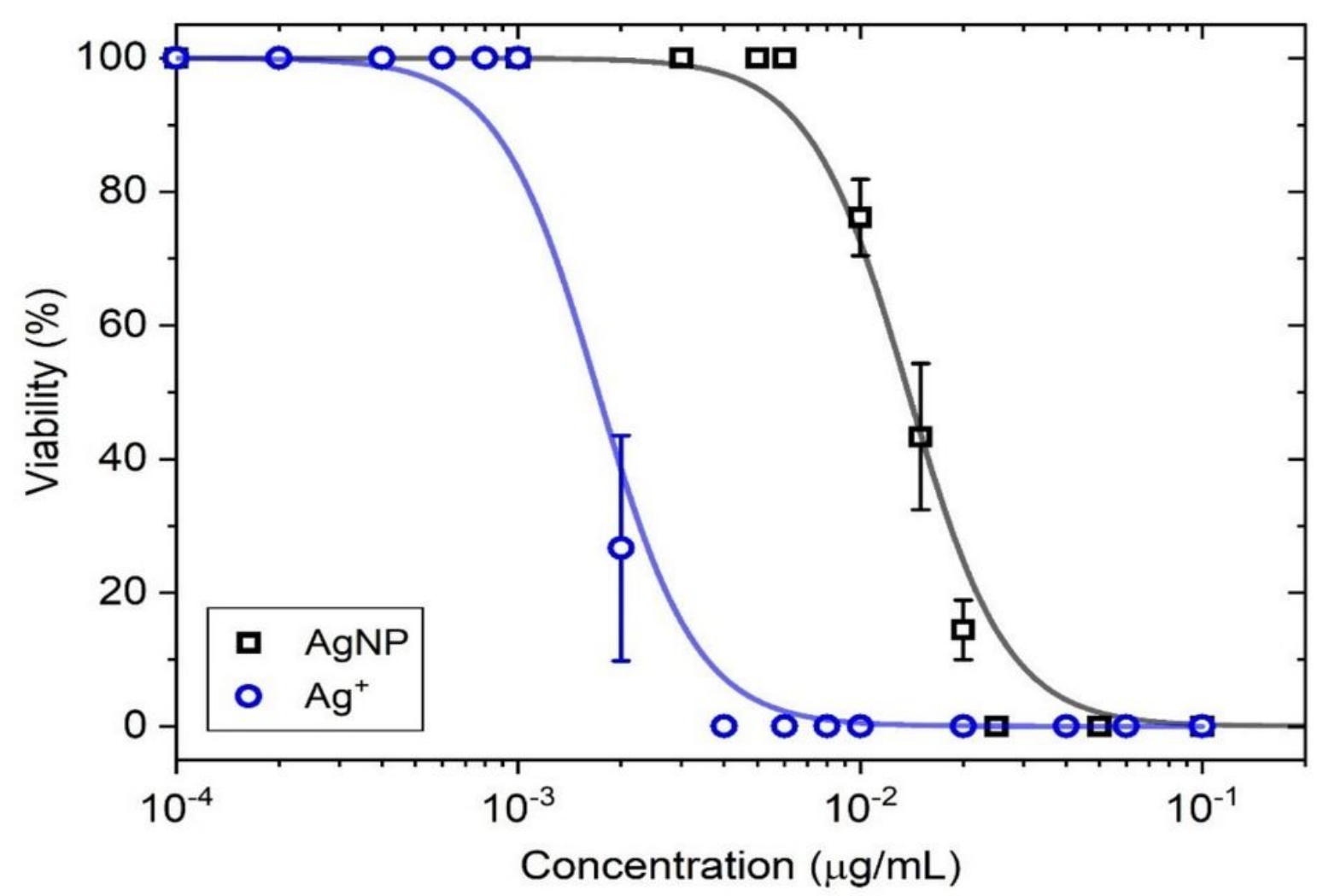

Figure 1

Viability of D. magna as a function of TiO2 and Ag nanoparticles (NPs) and Ag+. 

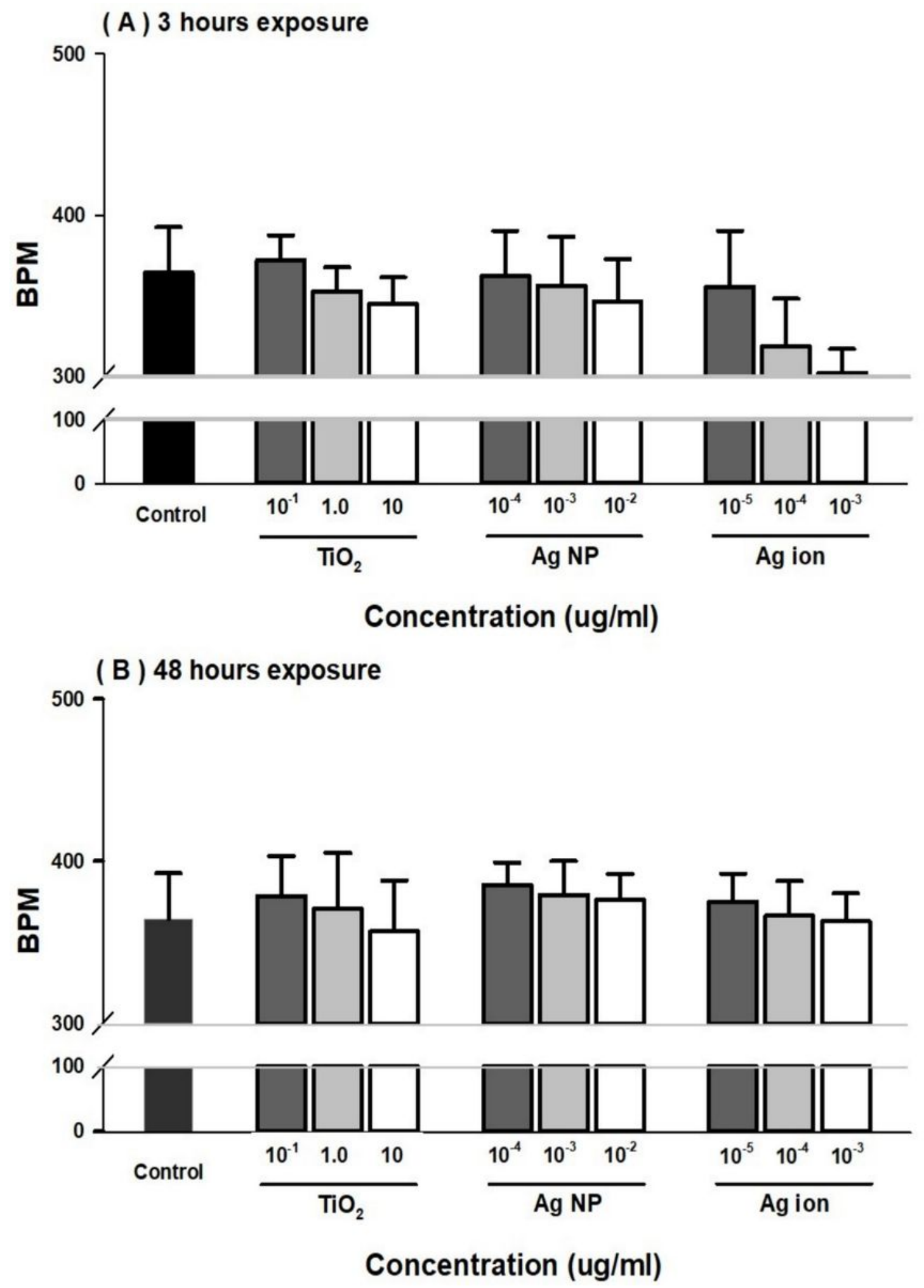

Figure 2

D. magna heart rate (BPM, beats per minute) changes after 3 and 48 hours of exposure to TiO2, AgNPs and $\mathrm{Ag}+$ at varying concentrations. Data are presented as the mean \pm standard deviation $(n=3)$. 


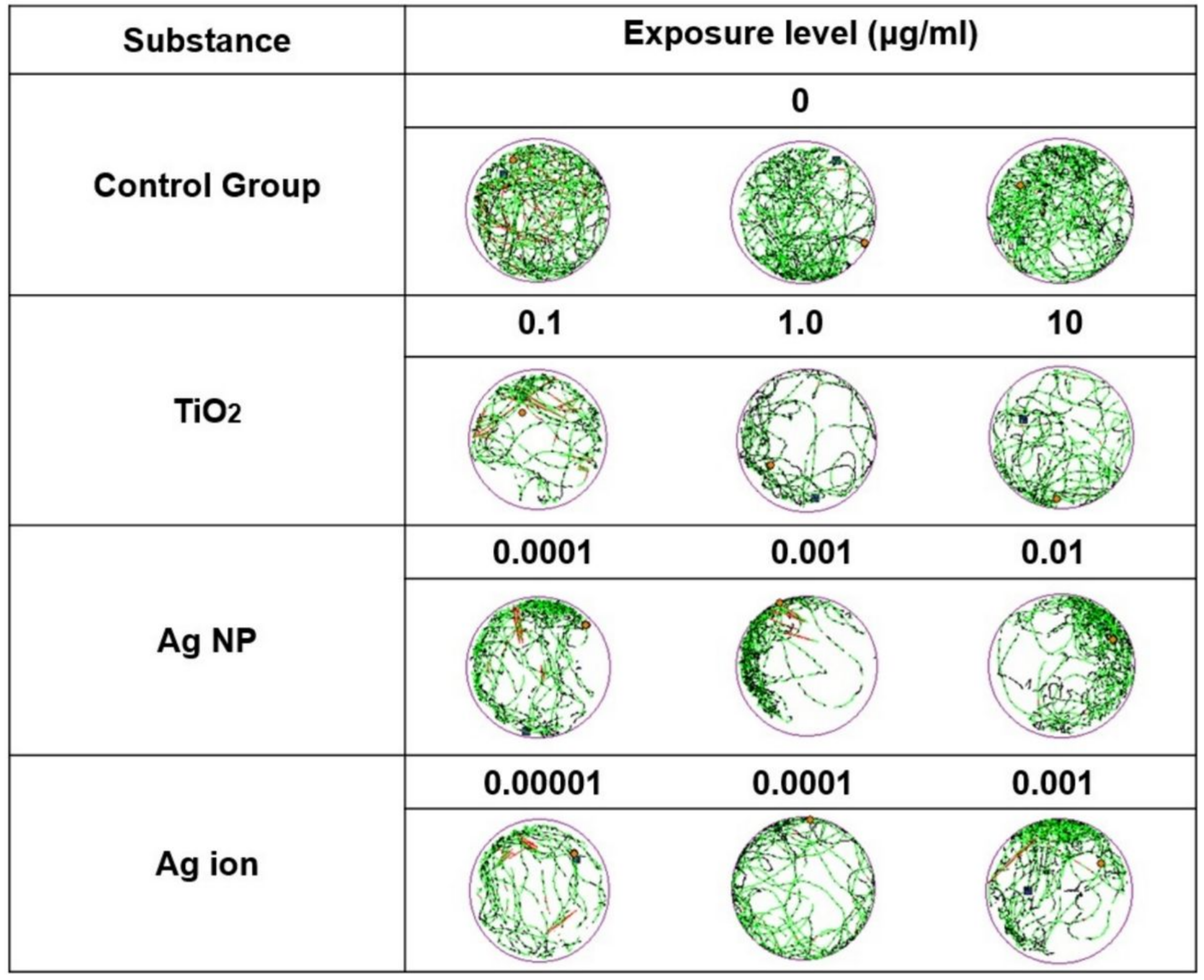

Figure 3

Representative images of the D. magna swimming paths for the initial 10 minutes as a function of the concentration of $\mathrm{TiO} 2, \mathrm{AgNPs}$ and $\mathrm{Ag}+$. 
( A ) $\mathrm{TiO}_{2}$

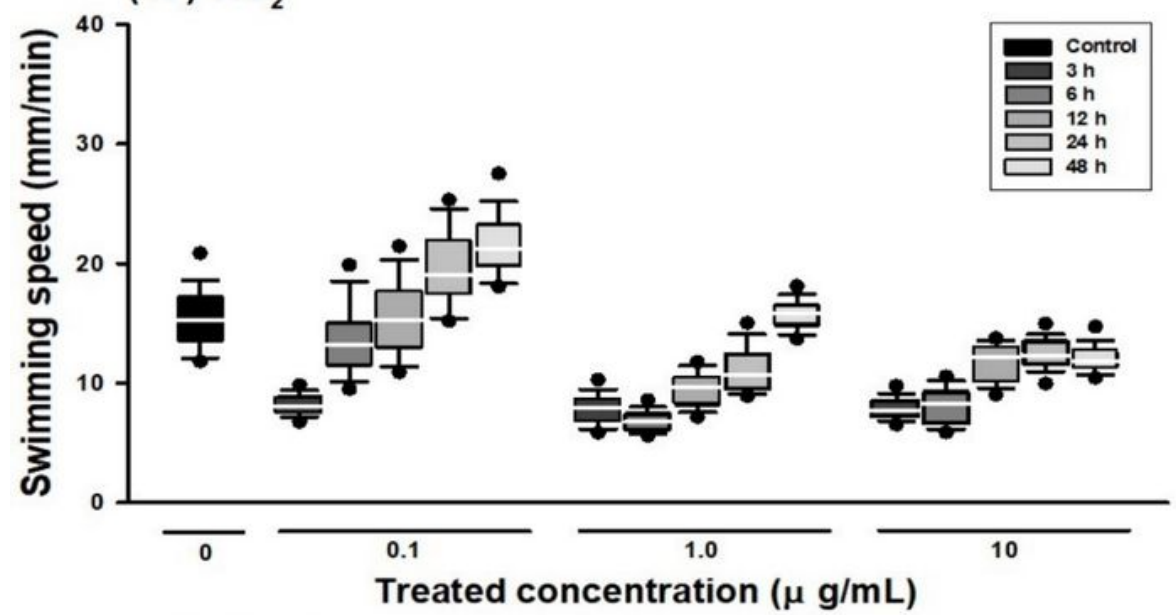

(B ) AgNP

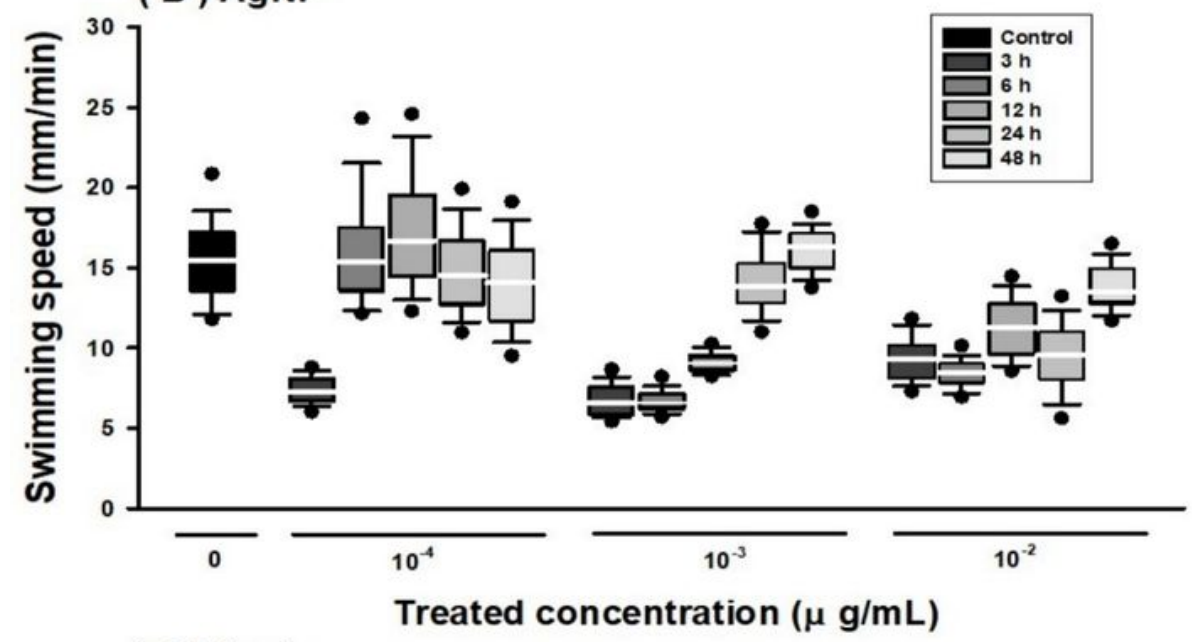

( C ) Ag +

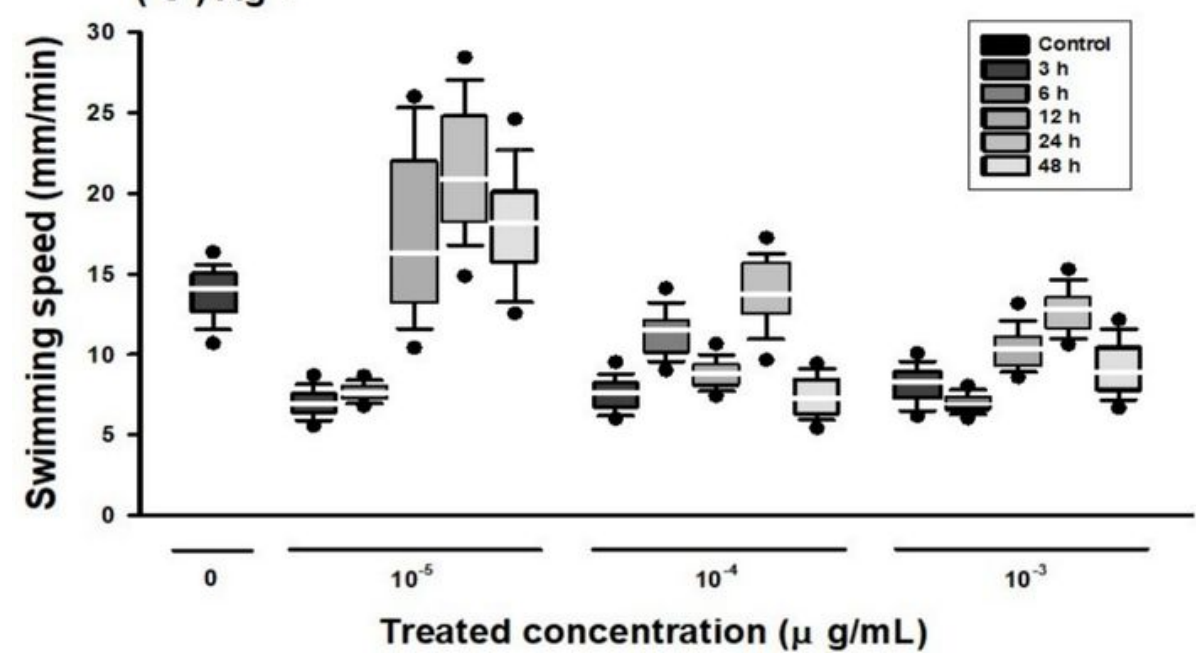

Figure 4

Plots of D. magna swimming speed as a function of the concentration of TiO2, AgNPs and Ag+ and exposure time (up to 48 hours). 


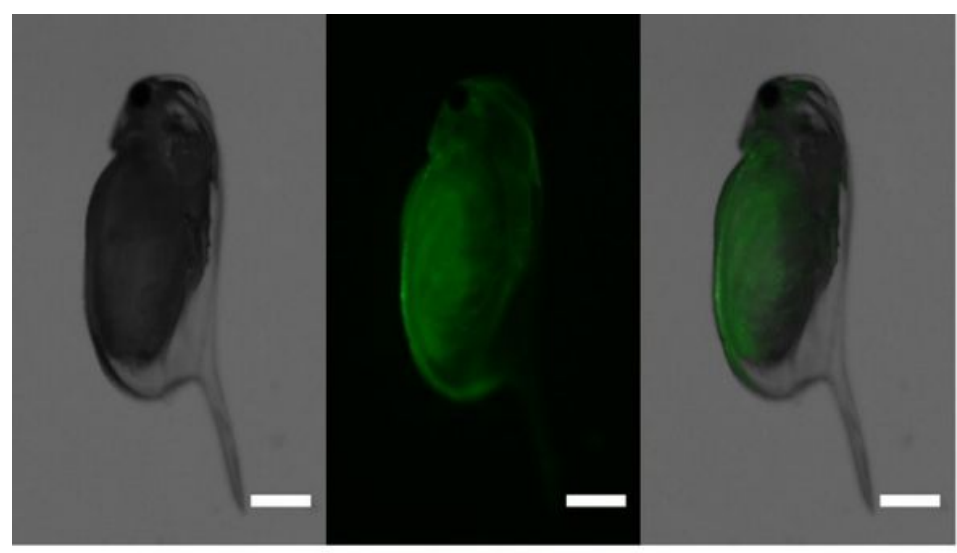

Control

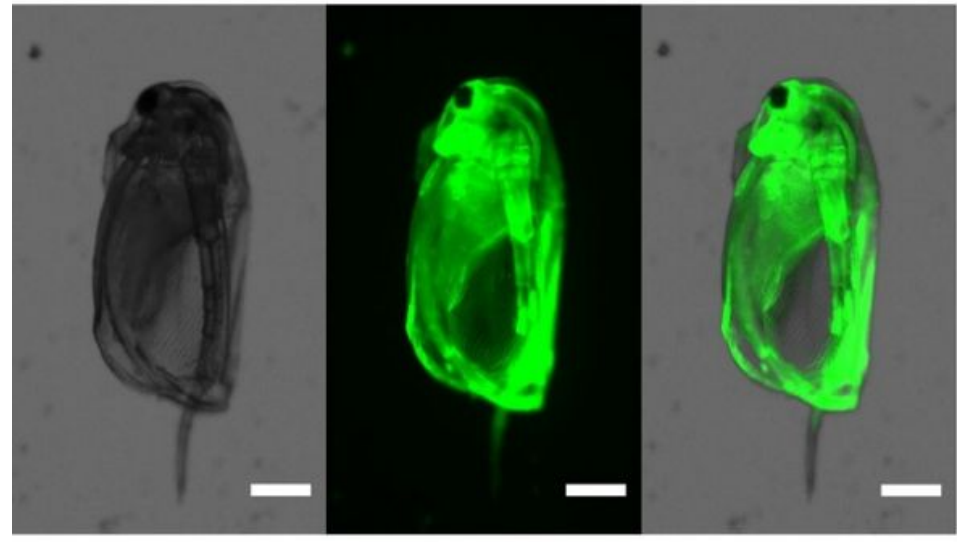

$\operatorname{Ag~NP}(0.001 \mu \mathrm{g} / \mathrm{mL})$

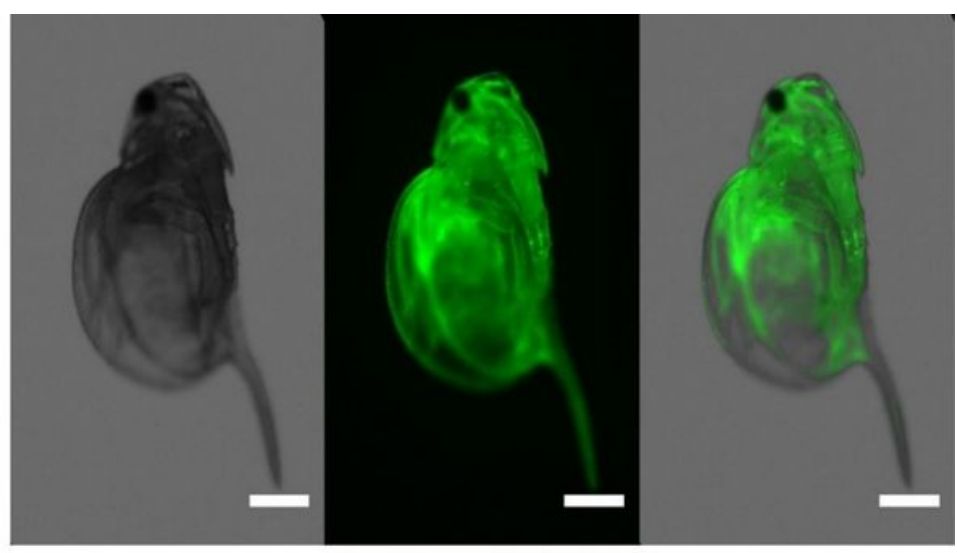

Ag ion $(0.0001 \mu \mathrm{g} / \mathrm{mL})$

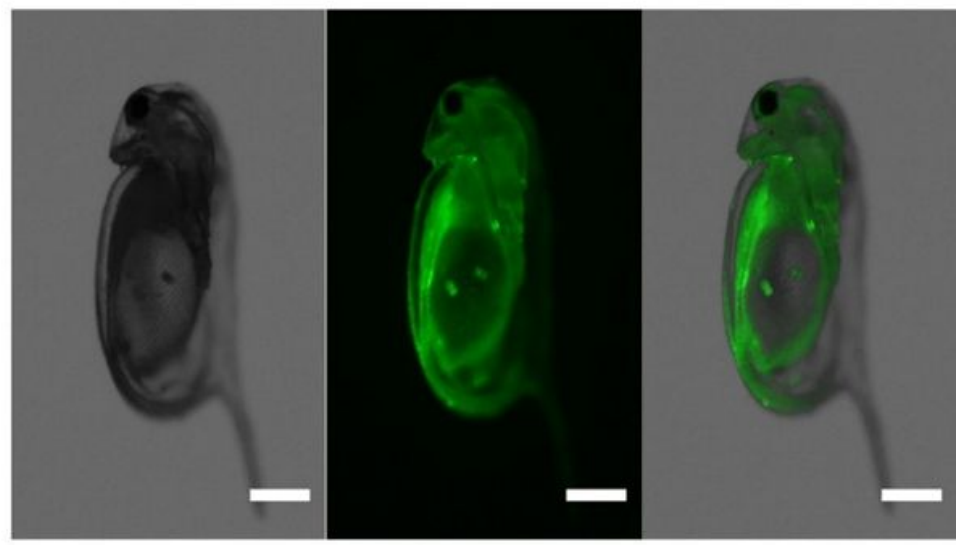

$\mathrm{TiO}_{2} \mathrm{NP}(10 \mu \mathrm{g} / \mathrm{mL})$

\section{Figure 5}

Representative optical microscopic images of D. manga exposed to Ag+, AgNPs, and TiO2 NPs for 3 hours. Each image set represents bright field, fluorescence, and merged images, from left to right. Scale bars, $200 \mu \mathrm{m}$. 

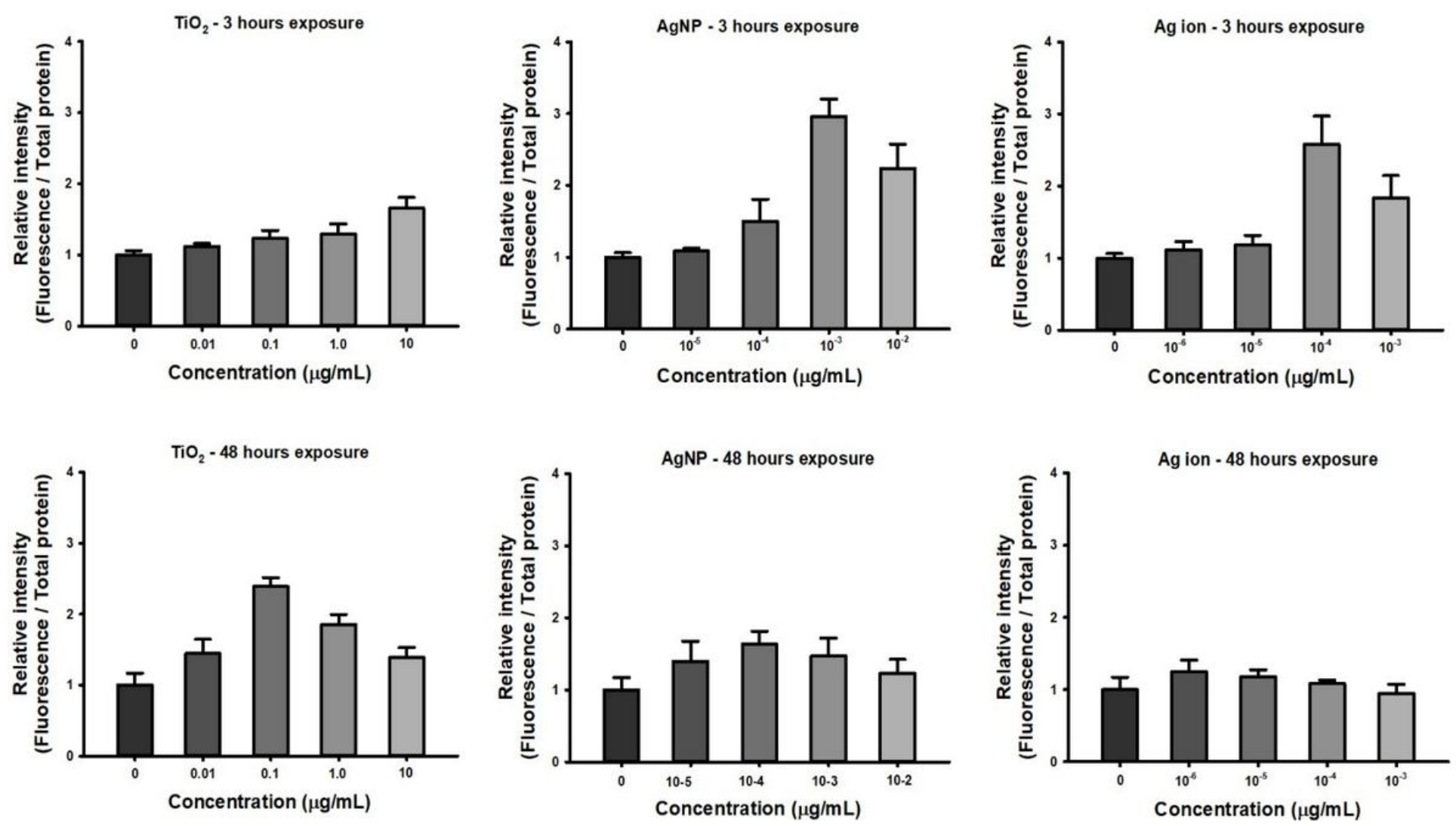

Figure 6

Effects of TiO2 and Ag NPs and Ag+ on reactive oxygen species (ROS) levels in D. magna after 3 and 48 hours of exposure Data are presented as the mean \pm standard deviation $(n=3)$. 


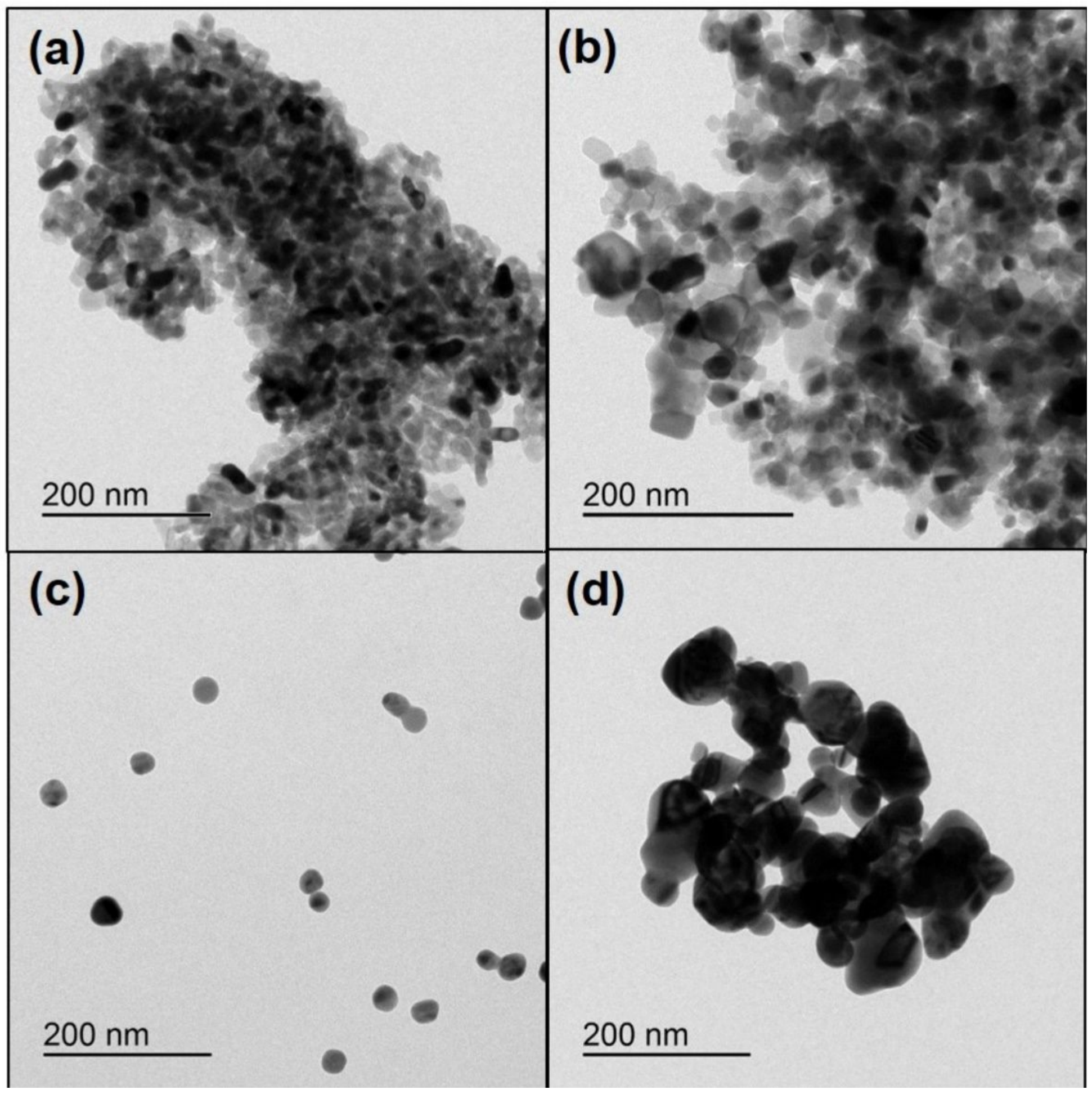

Figure 7

TEM images of the nanomaterials; (a) TiO2 NPs in distilled water. (b) TiO2 NPs in ISO medium. (c) AgNPs in distilled water. (d) AgNPs in ISO medium. Scale bars, $200 \mathrm{~nm}$.

\section{Supplementary Files}

This is a list of supplementary files associated with this preprint. Click to download. 
- Sci.Rep.SupplementaryInformationRev..docx 